\title{
ON QUASI-HYPERGEOMETRIC FUNCTIONS
}

\author{
Kazuhiko Aomoto and Kazumoto Iguchi
}

Dedicated to Richard Askey on the occasion of his 65th birthday

\begin{abstract}
We define quasi-hypergeometric functions of regular singular type and show that they are characterized by certain fractional differential equations on the one hand and by certain difference-differential equations on the other. Two examples of quasi-hypergeometric functions are given, namely quasi-algebraic functions and partition functions appearing in fractional exclusion statistics.
\end{abstract}

\section{Introduction}

L. Euler studied the Lambert series

$$
F(x)=\sum_{n=0}^{\infty}\left(\begin{array}{c}
\alpha+\beta n \\
n
\end{array}\right) x^{n},
$$

which is intimately related to the transcendental equation

$$
y-1=x y^{\beta},
$$

$[4,13]$. Recently, this kind of function has been given considerable attention by physicists. They play an important part in conformal field theory and fractional exclusion statistics. There is a pioneering work by B. Sutherland connecting them with fractional exclusion statistics and Calogero-Sutherland models [14-16]. The second author has extended some of these results to fractional exclusion statistics of multispecies of particles $[10,11]$, which is based on the results in $[8,18]$. This corresponds exactly to an extension of transcendental functions of the above type to multivariable ones.

In this note, we would like to generalize and give a mathematical background for these functions which we call "quasi-hypergeometric functions". These functions appear as an extension of general hypergeometric functions. The latter satisfy a holonomic system of differential equations of Barnes-Mellin type by means of $b$-functions [1-3]. A modern observation also has been discussed in relation to toric analysis by Gelfand et al. [5, 6].

However, the quasi-hypergeometric functions $F\left(x_{1}, \ldots, x_{n}\right)$ which we define here do not satisfy differential equations. We first present the system of fractional differential equations with respect to $x_{1}, \ldots, x_{n}$ which $F\left(x_{1}, \ldots, x_{n}\right)$ satisfy. Next, we show that $F\left(x_{1}, \ldots, x_{n}\right)$ also satisfies a kind of difference-differential equations with respect to $x_{1}, \ldots, x_{n}$ and other extra parameters $\alpha_{1}, \ldots, \alpha_{r} ; \alpha_{1}^{\prime}, \ldots, \alpha_{s}^{\prime}$ (analog of contiguous relations for hypergeometric functions).

Received March 30, 1998, revised June 9, 1998.

1991 Mathematics Subject Classification: 33E30, 32B30.

Key words and phrases: fractional exclusion statistics, quasi-hypergeometric functions. 
We can characterize these functions as the unique solutions to these functional equations.

\section{System of fractional differential equations}

Let $\alpha, \beta \in \mathbf{C}, \sigma=\left(\sigma_{1}, \ldots, \sigma_{n}\right), \sigma_{j}>0$ be given. We define a fractional derivative operator of order $-\beta$,

$$
P_{\sigma}(\alpha, \beta) f(x)=\frac{1}{\Gamma(\beta)} \int_{0}^{1} t^{\alpha-1}(1-t)^{\beta-1} f\left(t^{\sigma_{1}} x_{1}, \ldots, t^{\sigma_{n}} x_{n}\right) d t
$$

for a smooth function in a neighbourhood $\mathcal{U}$ of the origin of $\mathbf{C}^{n}$ (see [17]).

We assume that $\mathcal{U}$ is a Reinhaldt domain, i.e., $x=\left(x_{1}, \ldots, x_{n}\right) \in \mathcal{U}$ implies that $\left(\rho_{1} x_{1}, \ldots, \rho_{n} x_{n}\right) \in \mathcal{U}$ for arbitrary complex numbers $\rho_{j}$, such that $\left|\rho_{j}\right| \leq 1$.

If $\alpha$ and $\beta$ are positive, $P_{\sigma}(\alpha, \beta)$ is a well-defined operator, otherwise we may define it as a finite part of integrals at $t=0$ or $t=1$ in the sense of Hadamard [7].

The operator $P_{\sigma}(\alpha, \beta)$ is an operator reminiscent of the one which Humbert and Agarwal [9] defined for the function of Mittag-Leffler:

$$
E_{\beta}(x)=\sum_{n=0}^{\infty} \frac{1}{\Gamma(1+n \beta)} x^{n} .
$$

$P_{\sigma}(\alpha, \beta)$ satisfies the following basic properties.

Proposition 1. (i) For two arbitrary triples $(\alpha, \beta, \sigma)$ and $\left(\alpha^{\prime}, \beta^{\prime}, \sigma^{\prime}\right), P_{\sigma}(\alpha, \beta)$ $P_{\sigma^{\prime}}\left(\alpha^{\prime}, \beta^{\prime}\right)$ commute with each other, i.e.,

$$
P_{\sigma}(\alpha, \beta) \cdot P_{\sigma^{\prime}}\left(\alpha^{\prime}, \beta^{\prime}\right)=P_{\sigma^{\prime}}\left(\alpha^{\prime}, \beta^{\prime}\right) \cdot P_{\sigma}(\alpha, \beta) .
$$

(ii) $P_{\sigma}(\alpha, 0)$ is the identity operator.

Furthermore, if $\beta$ is a negative integer, say $\beta=-m, m=1,2,3, \ldots$, then $P_{\sigma}(\alpha,-m)$ reduces to a differential operator of order $m$,

$$
P_{\sigma}(\alpha,-m) f(x)=\prod_{k=1}^{m}\left(\alpha-k+\sum_{j=1}^{n} \sigma_{j} x_{j} \frac{\partial}{\partial x_{j}}\right) f(x) .
$$

For example,

$$
\begin{gathered}
P_{\sigma}(\alpha,-1) f(x)=\left(\alpha-1+\sum_{j=1}^{n} \sigma_{j} x_{j} \frac{\partial}{\partial x_{j}}\right) f(x) \\
P_{\sigma}(\alpha,-2) f(x)=\left[(\alpha-1)(\alpha-2)+\sum_{j=1}^{n}\left(2(\alpha-1) \sigma_{j}+\sigma_{j}^{2}\right) x_{j} \frac{\partial}{\partial x_{j}}\right. \\
\left.+\sum_{j, k=1}^{n} \sigma_{j} \sigma_{k} x_{j} x_{k} \frac{\partial^{2}}{\partial x_{j} \partial x_{k}}\right] f(x) .
\end{gathered}
$$

(iii)

$$
P_{\sigma}(\alpha+\beta,-\beta) \cdot P_{\sigma}(\alpha, \beta)=P_{\sigma}(\alpha, \beta) \cdot P_{\sigma}(\alpha+\beta,-\beta)=1
$$

so that $P_{\sigma}(\alpha+\beta,-\beta)$ can be regarded as the inverse of $P_{\sigma}(\alpha, \beta)$. 
(iv) For a monomial $x_{1}^{\nu_{1}} \cdots x_{n}^{\nu_{n}}$, we have

$$
P_{\sigma}(\alpha, \beta)\left(x_{1}^{\nu_{1}} \cdots x_{n}^{\nu_{n}} f(x)\right)=x_{1}^{\nu_{1}} \cdots x_{n}^{\nu_{n}} P_{\sigma}\left(\alpha+\sum_{j=1}^{n} \sigma_{j} \nu_{j}, \beta\right) f(x) .
$$

(v)

$$
\frac{\partial}{\partial x_{k}} P_{\sigma}(\alpha, \beta) f(x)=P_{\sigma}\left(\alpha+\sigma_{k}, \beta\right) \frac{\partial}{\partial x_{k}} f(x)
$$

(vi)

$$
P_{\sigma}(\alpha, \beta) x_{1}^{\nu_{1}} \cdots x_{n}^{\nu_{n}}=\frac{\Gamma\left(\alpha+\sum_{j=1}^{n} \sigma_{j} \nu_{j}\right)}{\Gamma\left(\alpha+\beta+\sum_{j=1}^{n} \sigma_{j} \nu_{j}\right)} x_{1}^{\nu_{1}} \cdots x_{n}^{\nu_{n}} .
$$

The proof of Proposition 1 is almost immediate except for (iii), which follows from the following lemma.

\section{Lemma 1.}

$$
P_{\sigma}(\alpha, \beta) \cdot P_{\sigma}\left(\alpha^{\prime}, \beta^{\prime}\right) f(x)=\int_{0}^{1} g(t) f\left(t^{\sigma_{1}} x_{1}, \ldots, t^{\sigma_{n}} x_{n}\right) \frac{d t}{t}
$$

where $g(s)$ denotes the Gauss hypergeometric function,

$$
g(s)=\frac{1}{\Gamma\left(\beta+\beta^{\prime}\right)}(1-s)^{\beta+\beta^{\prime}-1} s^{\alpha-\beta^{\prime}} F\left(\beta^{\prime}, \alpha^{\prime}+\beta^{\prime}-\alpha, \beta+\beta^{\prime} \mid \frac{s-1}{s}\right) .
$$

In particular, the following co-cycle property holds:

$$
\begin{aligned}
P_{\sigma}\left(\alpha+\beta^{\prime}, \beta\right) \cdot P_{\sigma}\left(\alpha, \beta^{\prime}\right) & =P_{\sigma}\left(\alpha, \beta^{\prime}\right) \cdot P_{\sigma}\left(\alpha+\beta^{\prime}, \beta\right) \\
& =P_{\sigma}\left(\alpha, \beta+\beta^{\prime}\right),
\end{aligned}
$$

which implies (iii) in Proposition 1 if $\beta+\beta^{\prime}=0$.

We now define a system of fractional differential equations (E) as follows.

Let $\alpha_{1}, \ldots, \alpha_{r}, \alpha_{1}^{\prime}, \ldots, \alpha_{s}^{\prime}$ be $r+s$ complex numbers, and $\beta_{i}=\left(\beta_{i j}\right)_{j=1}^{n} \in \mathbf{R}_{+}^{n}$ $(1 \leq i \leq r), \beta_{i}^{\prime}=\left(\beta_{i j}^{\prime}\right)_{j=1}^{n} \in \mathbf{R}_{+}^{n}(1 \leq i \leq s)$ be $r+s$ tuples of $n$-dimensional vectors with non-negative components.

We assume that the following relations hold. For each $j$,

$$
\sum_{i=1}^{s} \beta_{i j}^{\prime}=\sum_{i=1}^{r} \beta_{i j}+1
$$

This condition assures that the function $F(x)$ has tempered growth along a radial direction at the singularities, i.e., it has only a regular singularity.

We consider the following system of fractional differential equations for a function $F=F(x)$ depending on the variables $x_{1}, \ldots, x_{n}$ :

(E) $\frac{\partial}{\partial x_{j}} F=\prod_{i=1}^{s} P_{\beta_{i}^{\prime}}\left(\alpha_{i}^{\prime}+\beta_{i j}^{\prime},-\beta_{i j}^{\prime}\right) \cdot \prod_{i=1}^{r} P_{\beta_{i}}\left(\alpha_{i}, \beta_{i j}\right) F$

for $1 \leq j \leq n$. 
As is seen from (1), this system satisfies the compatibility condition

$$
\begin{aligned}
\frac{\partial}{\partial x_{j}}\left(\frac{\partial}{\partial x_{k}} F\right)= & \frac{\partial}{\partial x_{j}} \prod_{i=1}^{r} P_{\beta_{i}}\left(\alpha_{i}, \beta_{i k}\right) \prod_{i=1}^{s} P_{\beta_{i}^{\prime}}\left(\alpha_{i}^{\prime}+\beta_{i k}^{\prime},-\beta_{i k}^{\prime}\right) F \\
= & \prod_{i=1}^{r} P_{\beta_{i}}\left(\alpha_{i}+\beta_{i j}, \beta_{i k}\right) \prod_{i=1}^{s} P_{\beta_{i}^{\prime}}\left(\alpha_{i}^{\prime}+\beta_{i k}^{\prime}+\beta_{i j}^{\prime},-\beta_{i k}^{\prime}\right) \\
& \times \prod_{i=1}^{r} P_{\beta_{i}}\left(\alpha_{i}, \beta_{i j}\right) \prod_{i=1}^{s} P_{\beta_{i}^{\prime}}\left(\alpha_{i}^{\prime}+\beta_{i j}^{\prime},-\beta_{i j}^{\prime}\right) F \\
= & \prod_{i=1}^{r} P_{\beta_{i}}\left(\alpha_{i}, \beta_{i j}+\beta_{i k}\right) \cdot P_{\beta_{i}^{\prime}}\left(\alpha_{i}^{\prime}+\beta_{i j}^{\prime}+\beta_{i k}^{\prime},-\beta_{i j}^{\prime}-\beta_{i k}^{\prime}\right) F \\
= & \frac{\partial}{\partial x_{k}}\left(\frac{\partial}{\partial x_{j}} F\right)
\end{aligned}
$$

because of symmetry.

\section{Quasi-hypergeometric functions}

By using the parameters in the preceding section, we consider the following power series in $x$ at the origin.

$$
\begin{aligned}
F\left(\begin{array}{l}
\left\{\alpha_{1}^{\prime} ; \beta_{1}^{\prime}\right\}, \ldots,\left\{\alpha_{s}^{\prime} ; \beta_{s}^{\prime}\right\} \\
\left\{\alpha_{1} ; \beta_{1}\right\}, \ldots,\left\{\alpha_{r} ; \beta_{r}\right\}
\end{array} \mid x\right) \\
\quad=\sum_{\nu_{1}, \ldots, \nu_{n} \geq 0} \frac{\prod_{i=1}^{s} \Gamma\left(\alpha_{i}^{\prime}+\sum_{j=1}^{n} \beta_{i j}^{\prime} \nu_{j}\right)}{\prod_{i=1}^{r} \Gamma\left(\alpha_{i}+\sum_{j=1}^{n} \beta_{i j} \nu_{j}\right) \nu_{1} ! \cdots \nu_{n} !} x_{1}^{\nu_{1}} \cdots x_{n}^{\nu_{n}} .
\end{aligned}
$$

We first remark that the following lemma holds by Stirling's formula:

Lemma 2. We fix $a, b \in \mathbf{R}, k=1,2,3, \ldots$ Then, for a large positive number $t$, there exists a positive constant $C_{0}$ such that

$$
\frac{\Gamma(a+t)}{\Gamma\left(\frac{b+t}{k}\right)^{k}} \leq C_{0} t^{a-b+\frac{1}{2}(k-1)} k^{t} .
$$

As a consequence of this lemma, we have

Lemma 3. There exists a positive constant $C_{1}$ such that

$$
\begin{aligned}
& \left|\frac{\prod_{i=1}^{s} \Gamma\left(\alpha_{i}^{\prime}+\sum_{j=1}^{n} \beta_{i j}^{\prime} \nu_{j}\right)}{\prod_{i=1}^{r} \Gamma\left(\alpha_{i}+\sum_{j=1}^{n} \beta_{i j} \nu_{j}\right) \nu_{1} ! \cdots \nu_{n} !}\right| \\
& \leq C_{1}\left(\sum_{j=1}^{n} b_{j}^{\prime} \nu_{j}\right)^{\alpha_{1,2, \ldots, s}^{\prime}-\alpha_{1,2, \ldots, r}+\frac{1}{2}(-n+s-1)} \cdot(r+n)^{b_{1}^{\prime} \nu_{1}+\cdots+b_{n}^{\prime} \nu_{n}}
\end{aligned}
$$

where $\alpha_{1,2, \ldots, r}, \alpha_{1,2, \ldots, s}^{\prime}$ and $b_{j}, b_{j}^{\prime}$ denote the sums $\alpha_{1}+\cdots+\alpha_{r}, \alpha_{1}^{\prime}+\cdots+\alpha_{s}^{\prime}, \sum_{i=1}^{r} \beta_{i j}$ and $\sum_{i=1}^{s} \beta_{i j}^{\prime}$, respectively, such that $b_{j}^{\prime}=b_{j}+1$.

Proof. We assume that $\nu_{1}, \ldots, \nu_{n}$ are so large that $\alpha_{i}^{\prime}+\sum_{j=1}^{n} \beta_{i j}^{\prime} \nu_{j}>1$. 
We first note the inequality

$$
\begin{aligned}
& \left|\frac{\prod_{i=1}^{s} \Gamma\left(\alpha_{i}^{\prime}+\sum_{j=1}^{n} \beta_{i j}^{\prime} \nu_{j}\right)}{\prod_{i=1}^{r} \Gamma\left(\alpha_{1,2, \ldots, s}^{\prime}+\sum_{j=1}^{n} b_{j}^{\prime} \nu_{j}\right)}\right| \\
& =\int_{1 \geq t_{1}+\cdots+t_{s-1}, t_{j} \geq 0} t_{1}^{\alpha_{1}^{\prime}+\sum_{j=1}^{n} \beta_{1 j}^{\prime} \nu_{j}-1} \cdots t_{s-1}^{\alpha_{s-1}^{\prime}+\sum_{j=1}^{n} \beta_{s-1, j}^{\prime} \nu_{j}-1} \\
& \quad \quad \times\left(1-t_{1}-\cdots-t_{s-1}\right)^{\alpha_{s}^{\prime}+\sum_{j=1}^{n} \beta_{s j}^{\prime} \nu_{j}-1} d t_{1} \wedge \cdots \wedge d t_{s-1} \\
& \leq \\
& \quad \frac{1}{(s-1) !}
\end{aligned}
$$

since the integrand on the right-hand side is smaller than 1. have

On the other hand, by the log convexity of the Gamma function $\Gamma(x)$ for $x>0$, we

$$
\prod_{i=1}^{r} \Gamma\left(\alpha_{i}+\sum_{j=1}^{n} \beta_{i j} \nu_{j}\right) \nu_{1} ! \cdots \nu_{n} ! \geq \Gamma\left(\frac{\alpha_{1,2, \ldots, r}+n+\sum_{i=1}^{r} \sum_{j=1}^{n} b_{j}^{\prime} \nu_{j}}{n+r}\right)^{n+r} .
$$

These two inequalities imply Lemma 3 from Lemma 2.

As a consequence of Lemma 3, the series (4) converges in the polydisc $D$ defined by

$$
\left|x_{1}\right|<(r+n)^{-b_{1}^{\prime}}, \ldots,\left|x_{n}\right|<(r+n)^{-b_{n}^{\prime}}
$$

so that the function (4) defines a holomorphic function at the origin.

Furthermore, we have

Theorem 1. The function $F$ satisfies the equations (E) and can be characterized as the unique solution to (E) which is holomorphic at the origin and

$$
F(0)=\frac{\prod_{i=1}^{s} \Gamma\left(\alpha_{i}^{\prime}\right)}{\prod_{i=1}^{r} \Gamma\left(\alpha_{i}\right)}
$$

Proof. Assume that the holomorphic function at the origin

$$
F(x)=\sum_{\nu_{1} \geq 0, \ldots, \nu_{n} \geq 0} a_{\nu_{1}, \ldots, \nu_{n}} x_{1}^{\nu_{1}} \cdots x_{n}^{\nu_{n}}
$$

satisfies the equations (E). We fix $j$. From (vi) in Proposition 1, we have

$$
P_{\beta_{i}^{\prime}}\left(\alpha_{i}^{\prime}+\beta_{i j}^{\prime},-\beta_{i j}^{\prime}\right) x_{1}^{\nu_{1}} \cdots x_{n}^{\nu_{n}}=\frac{\Gamma\left(\alpha_{i}^{\prime}+\beta_{i j}^{\prime}+\sum_{k=1}^{n} \beta_{i k}^{\prime} \nu_{k}\right)}{\Gamma\left(\alpha_{i}^{\prime}+\sum_{k=1}^{n} \beta_{i k}^{\prime} \nu_{k}\right)} x_{1}^{\nu_{1}} \cdots x_{n}^{\nu_{n}}
$$

and

$$
P_{\beta_{i}}\left(\alpha_{i}, \beta_{i j}\right) x_{1}^{\nu_{1}} \cdots x_{n}^{\nu_{n}}=\frac{\Gamma\left(\alpha_{i}+\sum_{k=1}^{n} \beta_{i k} \nu_{k}\right)}{\Gamma\left(\alpha_{i}+\beta_{i j}+\sum_{k=1}^{n} \beta_{i k} \nu_{k}\right)} x_{1}^{\nu_{1}} \cdots x_{n}^{\nu_{n}} .
$$

The equations (E) give the recurrence relations with respect to $\nu_{1}, \nu_{2}, \ldots, \nu_{n}$ as

$$
\begin{aligned}
\left(\nu_{j}+1\right) a_{\nu_{1}, \ldots, \nu_{j}+1, \ldots, \nu_{n}}= & \prod_{i=1}^{r} \frac{\Gamma\left(\alpha_{i}+\sum_{k=1}^{n} \beta_{i k} \nu_{k}\right)}{\Gamma\left(\alpha_{i}+\beta_{i j}+\sum_{k=1}^{n} \beta_{i k} \nu_{k}\right)} . \\
& \times \prod_{i=1}^{s} \frac{\Gamma\left(\alpha_{i}^{\prime}+\beta_{i j}^{\prime}+\sum_{k=1}^{n} \beta_{i k}^{\prime} \nu_{k}\right)}{\Gamma\left(\alpha_{i}^{\prime}+\sum_{k=1}^{n} \beta_{i k}^{\prime} \nu_{k}\right)} \cdot a_{\nu_{1}, \ldots, \nu_{j}, \ldots, \nu_{n}} .
\end{aligned}
$$


These relations determine uniquely the coefficients $a_{\nu_{1}, \ldots, \nu_{j}, \ldots, \nu_{n}}$ except for a constant factor. If $a_{0, \ldots, 0}$ equals (5), then $F(x)$ coincides with

$$
F\left(\begin{array}{l}
\left\{\alpha_{1}^{\prime} ; \beta_{1}^{\prime}\right\}, \ldots,\left\{\alpha_{s}^{\prime} ; \beta_{s}^{\prime}\right\} \\
\left\{\alpha_{1} ; \beta_{1}\right\}, \ldots,\left\{\alpha_{r} ; \beta_{r}\right\}
\end{array} \mid x\right)
$$

Thus the theorem has been proved.

As a function of $\alpha_{1}, \ldots, \alpha_{r}, \alpha_{1}^{\prime}, \ldots, \alpha_{s}^{\prime}$ and $x$, the function

$$
F\left(\begin{array}{l}
\left\{\alpha_{1}^{\prime} ; \beta_{1}^{\prime}\right\}, \ldots,\left\{\alpha_{s}^{\prime} ; \beta_{s}^{\prime}\right\} \\
\left\{\alpha_{1} ; \beta_{1}\right\}, \ldots,\left\{\alpha_{r} ; \beta_{r}\right\}
\end{array} \mid x\right)
$$

is meromophic in $\alpha_{1}, \ldots, \alpha_{r}, \alpha_{1}^{\prime}, \ldots, \alpha_{s}^{\prime}$ in $\mathbf{C}^{r+s}$ and holomorphic in $x$ in the polydisc D.

When $\beta_{i j}$ and $\beta_{i j}^{\prime}$ are integers, the functions (4) are nothing more than general hypergeometric functions of Barnes-Mellin type.

\section{System of difference-differential equations}

In the preceding section, we have assumed that the parameters $\beta_{i j}$ are positive. This restriction is sometimes too restrictive.

In this section, we do not impose this condition on $\beta_{i j}$.

We consider a function $F=F\left(x ; \alpha ; \alpha^{\prime}\right)$ depending on the $(n+r+s)$ variables, $x=\left(x_{1}, \ldots, x_{n}\right), \alpha=\left(\alpha_{1}, \ldots, \alpha_{r}\right)$, and $\alpha^{\prime}=\left(\alpha_{1}^{\prime}, \ldots, \alpha_{s}^{\prime}\right)$.

We denote by $T_{\alpha_{i}}, T_{\alpha_{i}^{\prime}}$ the shift operators deriving from the displacements $\alpha_{i} \rightarrow \alpha_{i}+1, \alpha_{i}^{\prime} \rightarrow \alpha_{i}^{\prime}+1$,

$$
\begin{aligned}
& T_{\alpha_{i}} f\left(x ; \alpha_{1}, \ldots, \alpha_{i}, \ldots, \alpha_{r} ; \alpha^{\prime}\right)=f\left(x ; \alpha_{1}, \ldots, \alpha_{i}+1, \ldots, \alpha_{r} ; \alpha^{\prime}\right), \\
& T_{\alpha_{i}^{\prime}} f\left(x ; \alpha_{1}, \ldots, \alpha_{i}, \ldots, \alpha_{r} ; \alpha^{\prime}\right)=f\left(x ; \alpha ; \alpha_{1}^{\prime}, \ldots, \alpha_{i}^{\prime}+1, \ldots, \alpha_{s}^{\prime}\right),
\end{aligned}
$$

and also by $T_{\alpha_{i}}^{a}, T_{\alpha_{i}^{\prime}}^{a}$ the shift operators of the displacements $\alpha_{i} \rightarrow \alpha_{i}+a, \alpha_{i}^{\prime} \rightarrow \alpha_{i}^{\prime}+a$, respectively.

We consider the system of difference-differential equations $\left(\mathrm{E}^{*}\right)$ :

$$
\left(\mathrm{E}^{*}\right) \quad\left\{\begin{array}{rlrl}
F & =\left(\alpha_{i}+\sum_{k=1}^{n} \beta_{i k} x_{k} \frac{\partial}{\partial x_{k}}\right) T_{\alpha_{i}} F, & & 1 \leq i \leq r, \\
T_{\alpha_{i}^{\prime}} F & =\left(\alpha_{i}^{\prime}+\sum_{k=1}^{n} \beta_{i k}^{\prime} x_{k} \frac{\partial}{\partial x_{k}}\right) F, & 1 \leq i \leq s, \\
\frac{\partial}{\partial x_{j}} F=T_{\alpha_{1}}^{\beta_{1 j}} \cdots T_{\alpha_{r}}^{\beta_{r j}} \cdot T_{\alpha_{1}^{\prime}}^{\beta_{1 j}^{\prime}} \cdots T_{\alpha_{s}^{\prime}}^{\beta_{s j}^{\prime} F,} & & 1 \leq j \leq n .
\end{array}\right.
$$

Then we have the following theorem.

Theorem 2. The function (4) satisfies the equations $\left(\mathrm{E}^{*}\right)$. It is characterized as the unique solution to $\left(\mathrm{E}^{*}\right)$ which satisfies the initial condition

$$
F\left(0 ; \alpha ; \alpha^{\prime}\right)=\frac{\prod_{i=1}^{s} \Gamma\left(\alpha_{i}^{\prime}\right)}{\prod_{i=1}^{r} \Gamma\left(\alpha_{i}\right)} .
$$


Proof. The function (4) satisfies (7) and (8) because of the equalities

$$
\begin{aligned}
& T_{\alpha_{i}} \Gamma\left(\alpha_{i}+\sum_{k=1}^{n} \beta_{i k} \nu_{k}\right)=\left(\alpha_{i}+\sum_{k=1}^{n} \beta_{i k} \nu_{k}\right) \Gamma\left(\alpha_{i}+\sum_{k=1}^{n} \beta_{i k} \nu_{k}\right) \\
& T_{\alpha_{i}^{\prime}} \Gamma\left(\alpha_{i}^{\prime}+\sum_{k=1}^{n} \beta_{i k}^{\prime} \nu_{k}\right)=\left(\alpha_{i}^{\prime}+\sum_{k=1}^{n} \beta_{i k}^{\prime} \nu_{k}\right) \Gamma\left(\alpha_{i}^{\prime}+\sum_{k=1}^{n} \beta_{i k}^{\prime} \nu_{k}\right) .
\end{aligned}
$$

As for (9), we have

$$
\begin{aligned}
\frac{\partial}{\partial x_{j}} F(x) & =\sum_{\nu_{1}, \ldots, \nu_{n} \geq 0} \frac{\prod_{i=1}^{s} \Gamma\left(\alpha_{i}^{\prime}+\beta_{i j}^{\prime}+\sum_{k=1}^{n} \beta_{i k}^{\prime} \nu_{k}\right)}{\prod_{i=1}^{r} \Gamma\left(\alpha_{i}+\beta_{i j}+\sum_{k=1}^{n} \beta_{i k} \nu_{k}\right) \nu_{1} ! \cdots \nu_{n} !} x_{1}^{\nu_{1}} \cdots x_{n}^{\nu_{n}} \\
& =\text { the right-hand side of }(9) .
\end{aligned}
$$

Conversely, assume that $F(x)$ has the expansion (6) at the origin $x=0$ such that $a_{\nu_{1}, \ldots, \nu_{n}}=a_{\nu_{1}, \ldots, \nu_{n}}\left(\alpha, \alpha^{\prime}\right)$ depend on $\alpha, \alpha^{\prime}$ meromorphically. From (9), we have the recurrence relations

$$
\begin{aligned}
\left(\nu_{j}+1\right) a_{\nu_{1}, \ldots, \nu_{j}+1, \ldots, \nu_{n}}\left(\alpha, \alpha^{\prime}\right) & \\
& =a_{\nu_{1}, \ldots, \nu_{j}, \ldots, \nu_{n}}\left(\alpha_{1}+\beta_{1 j}, \ldots, \alpha_{r}+\beta_{r j} ; \alpha_{1}^{\prime}+\beta_{1 j}^{\prime}, \ldots, \alpha_{s}^{\prime}+\beta_{s j}^{\prime}\right),
\end{aligned}
$$

so that $a_{\nu_{1}, \ldots, \nu_{j}, \ldots, \nu_{n}}\left(\alpha, \alpha^{\prime}\right)$ are uniquely determined from $a_{0, \ldots, 0}\left(\alpha, \alpha^{\prime}\right)$.

The last one satisfies the difference equations from (7) and (8):

$$
T_{\alpha_{i}} a_{0, \ldots, 0}\left(\alpha, \alpha^{\prime}\right)=\alpha_{i}^{-1} a_{0, \ldots, 0}\left(\alpha, \alpha^{\prime}\right), \quad T_{\alpha_{i}^{\prime}} a_{0, \ldots, 0}\left(\alpha, \alpha^{\prime}\right)=\alpha_{i}^{\prime} a_{0, \ldots, 0}\left(\alpha, \alpha^{\prime}\right) .
$$

A general solution to these can be expressed as

$$
a_{0, \ldots, 0}\left(\alpha, \alpha^{\prime}\right)=\frac{\prod_{i=1}^{s} \Gamma\left(\alpha_{i}^{\prime}\right)}{\prod_{i=1}^{r} \Gamma\left(\alpha_{i}\right)} \cdot H\left(\alpha, \alpha^{\prime}\right)
$$

where $H\left(\alpha, \alpha^{\prime}\right)$ denotes an arbitrary periodic function with the periods 1 relative to each variable $\alpha_{i}, \alpha_{i}^{\prime}$.

In particular, if one takes $H\left(\alpha, \alpha^{\prime}\right)=1, F(x)$ coincides with

$$
F\left(\begin{array}{l}
\left\{\alpha_{1}^{\prime} ; \beta_{1}^{\prime}\right\}, \ldots,\left\{\alpha_{s}^{\prime} ; \beta_{s}^{\prime}\right\} \\
\left\{\alpha_{1} ; \beta_{1}\right\}, \ldots,\left\{\alpha_{r} ; \beta_{r}\right\}
\end{array} \mid x\right)
$$

We now fix a system of integers $\mathbf{l}=\left(l_{1}, \ldots, l_{r}\right)$ and $\mathbf{l}^{\prime}=\left(l_{1}^{\prime}, \ldots, l_{s}^{\prime}\right)$. We can take as $H\left(\alpha, \alpha^{\prime}\right)$ the periodic function

$$
H\left(\alpha, \alpha^{\prime}\right)=\exp \left[2 \pi i\left(\sum_{\mu=1}^{r} l_{\mu} \alpha_{\mu}+\sum_{\mu=1}^{s} l_{\mu}^{\prime} \alpha_{\mu}^{\prime}\right)\right]
$$

then we have the solution $F(x)$ to $\left(\mathrm{E}^{*}\right)$ which has the expression

$$
F(x)=\exp \left[2 \pi i\left(\sum_{\mu=1}^{r} l_{\mu} \alpha_{\mu}+\sum_{\mu=1}^{s} l_{\mu}^{\prime} \alpha_{\mu}^{\prime}\right)\right] F\left(\begin{array}{l}
\left\{\alpha_{1}^{\prime} ; \beta_{1}^{\prime}\right\}, \ldots,\left\{\alpha_{s}^{\prime} ; \beta_{s}^{\prime}\right\} \\
\left\{\alpha_{1} ; \beta_{1}\right\}, \ldots,\left\{\alpha_{r} ; \beta_{r}\right\}
\end{array} \mid x^{*}\right)
$$

where $x^{*}=\left(x_{1}^{*}, \ldots, x_{n}^{*}\right)$ denotes the point such that

$$
x_{1}^{*}=x_{1} \exp \left[2 \pi i\left(\sum_{\mu=1}^{r} l_{\mu} \beta_{\mu 1}+\sum_{\mu=1}^{s} l_{\mu}^{\prime} \beta_{\mu 1}^{\prime}\right)\right], \ldots, x_{n}^{*}=x_{n} \exp \left[2 \pi i\left(\sum_{\mu=1}^{r} l_{\mu} \beta_{\mu n}+\sum_{\mu=1}^{s} l_{\mu}^{\prime} \beta_{\mu n}^{\prime}\right)\right] \text {. }
$$


We shall abbreviate these solutions as $F_{11^{\prime}}(x)$,

Since an arbitrary periodic function $H\left(\alpha, \alpha^{\prime}\right)$ has a Fourier expansion by using a sequence (11), we can conclude the following.

Proposition 2. Every solution to $\left(\mathrm{E}^{*}\right)$ is a linear combination of a countable number of the solutions $F_{\mathbf{l}^{\prime}}(x)$.

\section{Examples}

Example 1. Let $\alpha_{1}, \alpha_{2} \in \mathbf{R}$ and $\beta_{1}, \beta_{2} \in \mathbf{R}_{+}$be given such that $\beta_{1}+\beta_{2}=1$. The function

$$
F=F\left(\left\{\alpha_{1} ; \beta_{1}\right\},\left\{\alpha_{2} ; \beta_{2}\right\} \mid x\right)=\sum_{n=0}^{\infty} \frac{\Gamma\left(\alpha_{1}+\beta_{1} n\right) \Gamma\left(\alpha_{2}+\beta_{2} n\right)}{n !} x^{n}
$$

converges in the disc $|x|<\beta_{1}^{-\beta_{1}} \beta_{2}^{-\beta_{2}}$ and satisfies the equation (E):

$$
\frac{d F}{d x}=P_{\beta_{1}}\left(\alpha_{1}+\beta_{1},-\beta_{1}\right) P_{\beta_{2}}\left(\alpha_{2}+\beta_{2},-\beta_{2}\right) F .
$$

The function $F\left(\left\{\alpha_{1} ; \beta_{1}\right\},\left\{\alpha_{2} \beta_{2}\right\} \mid x\right)$ is the unique solution to (E) which is holomorphic at the origin and such that $F(0)=\Gamma\left(\alpha_{1}\right) \Gamma\left(\alpha_{2}\right)$. It also satisfies the equation $\left(\mathrm{E}^{*}\right)$ :

$$
T_{\alpha_{1}} F=\left(\alpha_{1}+\beta_{1} x \frac{d}{d x}\right) F, \quad T_{\alpha_{2}} F=\left(\alpha_{2}+\beta_{2} x \frac{d}{d x}\right) F, \quad \frac{d}{d x} F=T_{\alpha_{1}}^{\beta_{1}} T_{\alpha_{2}}^{\beta_{2}} F .
$$

The equations $\left(\mathrm{E}^{*}\right)$ also are satisfied by the functions

$$
F_{l_{1}, l_{2}}(x)=\exp \left[2 \pi i\left(l_{1} \alpha_{1}+l_{2} \alpha_{2}\right)\right] F\left(\left\{\alpha_{1} ; \beta_{1}\right\},\left\{\alpha_{2} ; \beta_{2}\right\} \mid \exp \left[2 \pi i\left(l_{1} \beta_{1}+l_{2} \beta_{2}\right)\right] x\right)
$$

for all $\left(l_{1}, l_{2}\right) \in \mathbf{Z}^{2}$.

$F_{l_{1}, l_{2}}$ does not satisfy the equation (E) but instead satisfies

$$
\frac{d F}{d x}=\exp \left[2 \pi i\left(l_{1} \beta_{1}+l_{2} \beta_{2}\right)\right] P_{\beta_{1}}\left(\alpha_{1}+\beta_{1},-\beta_{1}\right) P_{\beta_{2}}\left(\alpha_{2}+\beta_{2},-\beta_{2}\right) F
$$

It is characterized as the unique solution to (13), which is holomorphic at the origin and $F(0)=\exp \left[2 \pi i\left(l_{1} \alpha_{1}+l_{2} \alpha_{2}\right)\right] \Gamma\left(\alpha_{1}\right) \Gamma\left(\alpha_{2}\right)$.

On the other hand, by using the equalities

$$
\frac{\Gamma\left(\alpha_{1}+\beta_{1} n\right) \Gamma\left(\alpha_{2}+\beta_{2} n\right)}{\Gamma\left(\alpha_{1}+\alpha_{2}+n\right)}=\int_{0}^{\infty} u^{\alpha_{1}+\beta_{1} n-1}(1+u)^{-\alpha_{1}-\alpha_{2}-n} d u
$$

and the binomial expansion

$$
\sum_{n=0}^{\infty} \frac{\Gamma\left(\alpha_{1}+\alpha_{2}+n\right)}{n !} x^{n}=\Gamma\left(\alpha_{1}+\alpha_{2}\right)(1-x)^{-\alpha_{1}-\alpha_{2}},
$$

we get the integral expression for $F(x)$ given by

$$
F(x)=\Gamma\left(\alpha_{1}+\alpha_{2}\right) \int_{0}^{\infty} u^{\alpha_{1}-1}\left(1+u-u^{\beta_{1}} x\right)^{-\alpha_{1}-\alpha_{2}} d u
$$

for $|x|<\beta_{1}^{-\beta_{1}} \beta_{2}^{-\beta_{2}}$. We simply denote the number $\beta_{1}^{-\beta_{1}} \beta_{2}^{-\beta_{2}}$ by $c$.

At $x=0$, the quasi-algebraic equation

$$
1+u-x u^{\beta_{1}}=0
$$

has the two particular solutions $u=u_{+}(x), u_{-}(x)$

$$
u_{+}(x)=-1+e^{\pi i \beta_{1}} x+\cdots, \quad u_{-}(x)=-1+e^{-\pi i \beta_{1}} x+\cdots,
$$


whose coefficients are complex conjugates of each other. When $x$ increases and approaches $c$, then $u_{ \pm}(x)$ move in the upper (lower) half plane and approach the positive number $\beta_{1} / \beta_{2}$. One sees that the function (15) has a singularity of the braid type at the point $x=c$.

Example 2. Let $\alpha_{1}, \alpha_{2}, \beta_{1}, \beta_{2} \in \mathbf{R}$ be given such that $\beta_{1}=\beta_{2}+1$.

Consider the function

$$
F=F\left(\begin{array}{l}
\left\{\alpha_{1} ; \beta_{1}\right\} \\
\left\{\alpha_{2} ; \beta_{2}\right\}
\end{array} \mid x\right)=\sum_{n=0}^{\infty} \frac{\Gamma\left(\alpha_{1}+\beta_{1} n\right)}{\Gamma\left(\alpha_{2}+\beta_{2} n\right) n !} x^{n}
$$

in the following two cases.

Case (i) $\beta_{1}>1, \beta_{2}>0 . F$ converges for $|x|<c, c=\beta_{1}^{-\beta_{1}}\left(\beta_{1}-1\right)^{\beta_{1}-1}$, and is the unique solution to

$$
\text { (E) } \frac{d F}{d x}=P_{\beta_{1}}\left(\alpha_{1}+\beta_{1},-\beta_{1}\right) P_{\beta_{2}}\left(\alpha_{2}, \beta_{2}\right) F
$$

such that $F(0)=\frac{\Gamma\left(\alpha_{1}\right)}{\Gamma\left(\alpha_{2}\right)} . F$ also satisfies the equations $\left(\mathrm{E}^{*}\right)$ :

$$
T_{\alpha_{1}} F=\left(\alpha_{1}+\beta_{1} x \frac{d}{d x}\right) F, \quad F=\left(\alpha_{2}+\beta_{2} x \frac{d}{d x}\right) T_{\alpha_{2}} F, \quad \frac{d}{d x} F=T_{\alpha_{1}}^{\beta_{1}} T_{\alpha_{2}}^{\beta_{2}} F .
$$

$F$ has the integral expression

$$
F=-\frac{\Gamma\left(\alpha_{1}+1-\alpha_{2}\right)}{2 \pi i} \int_{\mathcal{L}} w^{\alpha_{1}-1}\left(1-w+x w^{\beta_{1}}\right)^{-\alpha_{1}-1+\alpha_{2}} d w
$$

for $|x|<c$. Assume for simplicity that $0<x<c$. Then the path of integration $\mathcal{L}$ is constructed as follows. There exist two positive solutions $w_{1}, w_{2}$ to the equation

$$
1-w+x w^{\beta_{1}}=0
$$

such that $1<w_{1}<w_{2}$.

We construct a path $\mathcal{L}$ starting from 0 in the lower half plane, crossing the interval $\left[w_{1}, w_{2}\right]$ and going to 0 in the upper half plane.

When $x$ tends to $0, w_{1}$ approaches 1 , and the integral (17) is holomorphic in $x$ at $x=0$. On the other hand, when $x$ approaches $c$, then $w_{1}, w_{2}$ approach each other. Therefore, the integral (17) is no longer holomorphic at $x=c$. The function $F(x)$ then has a singularity of braid type there. In particular, if $\alpha_{1}=\alpha_{2}$, then $F$ reduces to

$$
F(x)=\sum_{n=0}^{\infty}\left(\begin{array}{c}
\alpha_{1}+\beta_{1} n \\
n
\end{array}\right) x^{n}=\frac{w_{1}^{\alpha_{1}}}{\beta_{1}+\left(1-\beta_{1}\right) w_{1}}
$$

which is a well-known formula [13].

Case (ii) $1>\beta_{1}>0,0>\beta_{2}>-1$.

By using the Gauss identity $\Gamma(\lambda) \Gamma(1-\lambda)=\pi / \sin \pi \lambda, F(x)$ can be written as

$$
F(x)=\sum_{n=0}^{\infty} \frac{\Gamma\left(\alpha_{1}+\beta_{1} n\right) \Gamma\left(1-\alpha_{2}-\beta_{2} n\right)}{n !} \frac{\sin \pi\left(\alpha_{2}+\beta_{2} n\right)}{\pi} x^{n}
$$


Hence, $F(x)$ can be written as $F=F_{+}-F_{-}$where

$$
\begin{aligned}
& F_{+}(x)=\frac{\exp \left[\pi i \alpha_{2}\right]}{2 \pi i} F\left(\left\{\alpha_{1} ; \beta_{1}\right\},\left\{1-\alpha_{2} ;-\beta_{2}\right\} \mid \exp \left[\pi i \beta_{2}\right] x\right) \\
& F_{-}(x)=\frac{\exp \left[-\pi i \alpha_{2}\right]}{2 \pi i} F\left(\left\{\alpha_{1} ; \beta_{1}\right\},\left\{1-\alpha_{2} ;-\beta_{2}\right\} \mid \exp \left[-\pi i \beta_{2}\right] x\right) .
\end{aligned}
$$

Each of them satisfies the equations of the type (14) which are different from each other. But both of them satisfy the same equations $\left(E^{*}\right)$ and (16).

\section{Example 3.}

$$
F=\sum_{n=0}^{\infty} \frac{\prod_{k=1}^{s} \Gamma\left(\alpha_{k}^{\prime}+\beta_{k}^{\prime} n\right)}{\prod_{k=1}^{r} \Gamma\left(\alpha_{k}+\beta_{k} n\right) n !} x^{n}
$$

for $\beta_{k}, \beta_{k}^{\prime}>0$ and with the relation $\beta_{1}+\cdots \beta_{r}+1=\beta_{1}^{\prime}+\cdots+\beta_{s}^{\prime}$, is a general one in the single variable case. In a way similar to Examples 1 and 2 , one can show that the above series is convergent for $|x|<c$ for $c=\beta_{1}^{\beta_{1}} \cdots \beta_{r}^{\beta_{r}} \cdot \beta_{1}^{\prime-\beta_{1}^{\prime}} \cdots \beta_{s}^{\prime-\beta_{s}^{\prime}}$.

$F$ satisfies the equations

$$
\begin{aligned}
& \frac{d}{d x} F=\prod_{k=1}^{r} P_{\beta_{k}}\left(\alpha_{k}, \beta_{k}\right) \prod_{k=1}^{s} P_{\beta_{k}^{\prime}}\left(\alpha_{k}^{\prime}+\beta_{k}^{\prime},-\beta_{k}^{\prime}\right) F, \\
& \left\{\begin{array}{rlrl}
F & =\left(\alpha_{k}+\beta_{k} x \frac{d}{d x}\right) T_{\alpha_{k}} F, & & 1 \leq k \leq r, \\
T_{\alpha_{k}^{\prime}} F & =\left(\alpha_{k}^{\prime}+\beta_{k}^{\prime} x \frac{d}{d x}\right) F, & 1 \leq k \leq s, \\
\frac{d}{d x} F & =T_{\alpha_{1}}^{\beta_{1}} \cdots T_{\alpha_{r}}^{\beta_{r}} T_{\alpha_{1}^{\prime}}^{\beta_{1}^{\prime}} \cdots T_{\alpha_{s}^{\prime}}^{\beta_{s}^{\prime}} F . &
\end{array}\right.
\end{aligned}
$$

We will show in a subsequent article that $F$ has a singularity at $x=c$ and has a power series expansion near $c$, namely,

$$
F(x)=(c-x)^{\delta}\left[a_{0}+a_{1}(c-x)+a_{2}(c-x)^{2}+\cdots\right]+(\text { a holomorphic function) }
$$

where $\delta$ denotes $\delta=\alpha_{1,2, \ldots, r}-\alpha_{1,2, \ldots, s}^{\prime}+\frac{1}{2}(s-r-1)$.

In view of (iii) in Proposition $1,(\mathrm{E})$ is equivalent to

$$
\prod_{k=1}^{r} P_{\beta_{k}}\left(\alpha_{k}+\beta_{k},-\beta_{k}\right) \frac{d}{d x} F=\prod_{k=1}^{s} P_{\beta_{k}^{\prime}}\left(\alpha_{k}^{\prime}+\beta_{k}^{\prime},-\beta_{k}^{\prime}\right) F
$$

When $\beta_{k}=\beta_{k}^{\prime}=1$ for all $k, s$ must be equal to $r+1$. $F(x)$ reduces to the hypergeometric function of higher order $[2,3]$

$$
{ }_{r} F_{r+1}\left(\begin{array}{c|c}
\alpha_{1}^{\prime}, \ldots, \alpha_{r+1}^{\prime} \\
\alpha_{1}, \ldots, \alpha_{r}
\end{array}\right)
$$

(E) reduces to the ordinary differential equation

$$
\prod_{k=1}^{r}\left(\alpha_{k}+x \frac{d}{d x}\right) \frac{d}{d x} F=\prod_{k=1}^{r+1}\left(\alpha_{k}^{\prime}+x \frac{d}{d x}\right) F .
$$

Example 4. We fix $\lambda_{0}, \lambda_{1}, \ldots, \lambda_{n} \in \mathbf{R}$. The quasi-algebraic equation with respect to $y$ given by

$$
y^{\lambda_{0}}+x_{1} y^{\lambda_{1}}+\cdots+x_{n} y^{\lambda_{n}}-1=0
$$


has a holomorphic solution in $\left(x_{1}, \ldots, x_{n}\right)$ at the origin such that $y=1$ for $x=0$. Then, for an arbitrary $\rho \in \mathbf{C}, y^{\rho}$ has the expansion in $x$,

$$
y^{\rho}=\frac{\rho}{\lambda_{0}} \sum_{\nu_{1} \geq 0, \ldots, \nu_{n} \geq 0}(-1)^{|\nu|} \frac{\Gamma\left(A_{\nu}\right)}{\Gamma\left(A_{\nu}-|\nu|+1\right) \nu_{1} ! \cdots \nu_{n} !} x_{1}^{\nu_{1}} \cdots x_{n}^{\nu_{n}}
$$

where $A_{\nu}=\frac{1}{\lambda_{0}}\left(\rho+\lambda_{1} \nu_{1}+\cdots+\lambda_{n} \nu_{n}\right)$ and $|\nu|=\nu_{1}+\cdots+\nu_{n}$, i.e.,

$$
y^{\rho}=\frac{\rho}{\lambda_{0}} F\left(\begin{array}{c|c}
\left\{\frac{\rho}{\lambda_{0}} ;\left(\frac{\lambda_{1}}{\lambda_{0}}, \ldots, \frac{\lambda_{n}}{\lambda_{0}}\right)\right\} \\
\left\{\frac{\rho}{\lambda_{0}}+1 ;\left(\frac{\lambda_{1}}{\lambda_{0}}-1, \ldots, \frac{\lambda_{n}}{\lambda_{0}}-1\right)\right\}
\end{array} \mid x_{1}, \ldots, x_{n}\right) .
$$

In fact, assume that $y^{\rho}$ has an expansion

$$
y^{\rho}=1+\sum_{\nu_{1} \geq 0, \ldots, \nu_{n} \geq 0,|\nu|>0} a_{\nu_{1}, \ldots, \nu_{n}} x_{1}^{\nu_{1}} \cdots x_{n}^{\nu_{n}} .
$$

Then, by the Cauchy integral formula, we have for a small positive number $\epsilon$,

$$
a_{\nu_{1}, \ldots, \nu_{n}}=\left(\frac{1}{2 \pi i}\right)^{n} \int_{\left|x_{1}\right|=\epsilon, \ldots,\left|x_{n}\right|=\epsilon} y^{\rho} x_{1}^{-\nu_{1}-1} \cdots x_{n}^{-\nu_{n}-1} d x_{1} \wedge \cdots \wedge d x_{n} .
$$

The change of variables of integration, $\left(x_{1}, \ldots, x_{n}\right) \rightarrow\left(x_{1}, \ldots, x_{n-1}, y\right)$, gives

$$
\begin{aligned}
a_{\nu_{1}, \ldots, \nu_{n}}=\left(\frac{1}{2 \pi i}\right)^{n} \int_{\left|x_{1}\right|=\epsilon, \ldots,\left|x_{n-1}\right|=\epsilon,|y-1|=\epsilon} y^{\rho} x_{1}^{-\nu_{1}-1} & \cdots x_{n}^{-\nu_{n}-1} \\
& \times \frac{\partial x_{n}}{\partial y} d x_{1} \wedge \cdots \wedge d x_{n-1} \wedge d y
\end{aligned}
$$

for $x_{n}=\left(1-y^{\lambda_{0}}-x_{1} y^{\lambda_{1}}-\cdots-x_{n-1} y^{\lambda_{n-1}}\right) / y^{\lambda_{n}}$.

Using the binomial expansion $y^{\rho}=\sum_{l=0}^{\infty}\left(\begin{array}{l}\rho \\ l\end{array}\right)(y-1)^{l}, a_{\nu_{1}, \ldots, \nu_{n}}$ is evaluated by residue calculus as in (18).

It has been known since $\mathrm{H}$. Mellin that if we put $\lambda_{0}=n+1, \lambda_{1}=n, \ldots, \lambda_{n}=1$, then $y$ reduces to a general algebraic function corresponding to the singularity of the A-type root system (see [3, 12], etc.).

Example 5. Consider the function

$$
F(x)=\sum_{\nu_{1}, \ldots, \nu_{n} \geq 0} \frac{\prod_{i=1}^{n} \Gamma\left(\alpha_{i}+\sum_{j=1}^{n} \beta_{i j}^{\prime} \nu_{j}\right)}{\prod_{i=1}^{n} \Gamma\left(\alpha_{i}+\sum_{j=1}^{n} \beta_{i j} \nu_{j}\right) \nu_{1} ! \cdots \nu_{n} !} x_{1}^{\nu_{1}} \cdots x_{n}^{\nu_{n}}
$$

where we assume $\beta_{i j}^{\prime}=\beta_{i j}=-g_{i j}$ for $i \neq j$ and $\beta_{i i}^{\prime}=\beta_{i i}+1=1-g_{i i}$ for suitable real numbers $g_{i j}$.

This function has been investigated in recent papers on statistical mechanics [10, 11 ] by the second author. It is described simply by using the function $w_{1}^{\alpha_{1}} \cdots w_{n}^{\alpha_{n}}$ depending on $x_{1}, \ldots, x_{n}$, which is derived from a system of the quasi-algebraic equations

$$
w_{i}=1+x_{i} w_{i}^{1-g_{i i}} w_{1}^{-g_{i 1}} \cdots w_{n}^{-g_{i n}} \quad 1 \leq i \leq n .
$$

These are the fundamental equations discovered by $\mathrm{Wu}$ [18] for describing mutual fractional exclusion statistics following Haldane [8] which is an extension of an earlier work by Sutherland [15] in the one variable case. The equations (20) can be solved explicitly as a power series expansion in $x_{1}, \ldots, x_{n}$ by the Lagrange inversion formula in the multivariable case, see [11] for details.

It seems an interesting problem to study the singularities and the monodromy property of $F(x)$ when $F(x)$ is analytically continued. 
Recently Prof. V. S. Retakh pointed out to us that quasi-hypergeometric functions are very similar to the GG-functions defined by Gelfand and Graev [19]. They seem to obtain an equivalent form of our equations $\left(\mathrm{E}^{*}\right)$, although we have not yet shown this. They define GG-functions as a wider class of functions which are not necessarily of regular singular type. For geometric reasons, we only consider here quasi-hypergeometric functions of regular singular type.

Acknowledgment. We would like to acknowledge a valuable comment by A. Gyoja.

\section{References}

1. K. Aomoto and M. Kita, Theory of Hypergeometric Functions, Springer-Verlag, Tokyo, 1994. (in Japanese).

2. P. Appell and J. Kampé de Feriét, Fonctions Hypérgéométriques et Hypérsphériques, Polynomes d'Hermite, Gauthiers-Villars, Paris, 1926.

3. G. Bellardinelli, Fonctions Hypérgéométriques de Plusieures Variables et Resolutions Analytiques des Equations Algébriques Générales, Paris, Gauthiers Villars, 1960.

4. B. C. Berndt, Ramanujan's Notebooks, Springer-Verlag, 1985.

5. I. M. Gelfand, M. I. Graev, and V. S. Retah, General hypergeometric systems of equations and series of hypergeometric type, Russian Math. Surveys 47 (1992), 1-88.

6. I. M. Gelfand, M. I. Graev, and A. V. Zelevinsky, Holonomic systems and series of hypergeometric type, Soviet Math. Dokl. 36 (1988), 5-10.

7. J. Hadamard, Lectures on Cauchy's Problem in Linear Partial Differential Equations, Dover, New York, 1952.

8. F. D. M. Haldane, "Fractional statistics" in arbitrary dimensions: a generalization of the Pauli principle, Phys. Rev. Lett. 67 (1991), 937-940.

9. P. Humbert and R. P. Agarwal, Sur la fonction de Mittag-Leffler et quelques-unes de ses généralisations, Bull. Sci. Math. 77 (1953), 180-185.

10. K. Iguchi, How does Lagrange's theorem solve thermodynamics of a multispecies quasiparticle gas with mutual fractional exclusion statistics? Modern Physics Letters B 11 (1997), 765-772.

11. __ Generalized Lagrange's theorem and thermodynamics of a multispecies quasiparticle gas with mutual fractional exclusion statistics, Phys. Rev. B. 58 (1998), 6892-6911.

12. S. Ishiura and M. Noumi, A calculus of the Gauss-Manin system of type $A_{l} I$, II, Proc. Japan Acad. 58(A) (1982), 13-16; 62-65.

13. G. Polya and G. Szegö, Problems and Theorems in Analysis I, Springer-Verlag, Berlin, 1972.

14. B. Sutherland, Quantum many problem in one dimension: Ground state, Jour. Math. Phys. 12 (1971), 246-250.

15. — , Quantum many problem in one dimension: Thermodynamics, Jour. Math. Phys. 12 (1971), 251-256.

16. ㄴ Exact results for a quantum many problem in one dimension, Phys. Rev. A 4 (1971), 2019-2021.

17. S. G. Samko, A. A. Kilbas, and O. L. Marichev, Fractional Integrals and Derivatives. Theory and Applications, Nauka i Technika, Minsk, 1987; English transl. Gordon and Breach, 1993.

18. Y.-S. Wu, Statistical distribution for generalized ideal gas of fractional statistical particles, Phys. Rev. Lett. 73 (1994), 922-925.

19. I. M. Gelfand and M. I. Graev, GG-functions and their relation to general hypergeometric functions, Russian Math. Surveys 52, 639-684.

(Aomoto) Graduate School of Mathematics, Nagoya University, Chimusa, NagoYa, 464-01, JAPAN

(Iguchi) 70-3, SiNHARI, HARIMACHI, ANAN, JAPAN 